\title{
Die 100 ersten Tage - Berufsanfängerinnen berichten
}

Denjenigen unter uns jungen Juristinnen, die sich noch in der Ausbildung befinden, wurden im Studium bereits die theoretischen Grundlagen der Rechtspraxis sowie eine wahlfachbezogene Vertiefung derselben vermittelt und die Gelegenheit gegeben, im Referendariat in die verschiedenen Bereiche der Rechtspraxis hineinzuschnuppern. Von der „theoretischen Praxis“ haben wir dadurch bereits eine gewisse Vorstellung, die sicherlich den Sprung in die „echte Praxis“ erleichtert. Dennoch birgt dieser Schritt in die Berufspraxis viele ungewohnte Herausforderungen, auf die selbst das Referendariat keine hinreichende Vor- bereitung leistet (und wohl auch nicht leisten kann). Spannend fanden wir daher den Rückblick auf die Berufsanfangsphase durch diejenigen von uns, die bereits erste praktische Erfahrungen gesammelt haben. Weil es heute so viele spannende juristische Berufsfelder gibt, wird dieser Beitrag in einem zweiten Teil in djbZ 4-2009 fortgesetzt. Im vorliegenden ersten Teil berichten zwei Juristinnen aus den tradierten juristischen Berufen (Rechtsanwältin, Richterin), im zweiten Teil kommen Berufsanfängerinnen in alternativen juristischen Berufen zu Wort.

\section{Aller Anfang ist spannend: Rechtsanwältin}

Diesen Artikel zum Berufseinstieg schreibe ich nun als Rechtsanwältin aus der Sicht einer Anwältin mit einem Jahr Anwaltszulassung. Wie war das „damals“ noch gleich?

Ach ja, da war doch was. Zweites Staatsexamen, mündliche Prüfung, nicht die gewünschte Note erhalten und einen Berg Stellenangebote vor mir. Den Leserinnen möchte ich die erste Phase meiner Bewerbungsaktivitäten sowie meine Erfahrungen mit der Arbeitsagentur ersparen. Nur so viel: Man wird besser im Laufe der Zeit.

Letztendlich suchte ich ein halbes Jahr nach dem richtigen Job. Den fand ich nun in meiner jetzigen Kanzlei, in der es mir von Anfang an gut gefallen hat. Da ich schon immer den Rechtsanwältinnenjob machen wollte (und nichts anderes, auch wenn man als Frau zum Staat soll, wie so viele versuchten, mir nahezulegen), füllt(e) mich diese Aufgabe von da an ganz aus. Ich wurde freie Mitarbeiterin in einer Kanzlei, die es seit zwei Jahren gab und die sich mittlerweile vor Aufträgen nicht mehr retten konnte und dringend Verstärkung brauchte. Kurios und eher selten an dieser Kanzlei ist, dass wir drei Rechtsanwältinnen sind und nur ein aktiver Rechtsanwalt. Das Geschlechterverhältnis ist bei uns umgekehrt zu anderen Kanzleien. Dies sprach bei mir übrigens für den Job. Da man als freie Mitarbeiterin selbstständig ist, brauchte ich einige Bedenkzeit. Zunächst war ich nicht sicher, ob ich mir die Selbstständigkeit vorstellen konnte. Dennoch handelte ich nach dem Motto „No risk, no fun“ und sagte zu. $\mathrm{Zu}$ diesem Zeitpunkt war ich übrigens schon als Rechtsanwältin zugelassen und konnte somit sofort loslegen.

Nun ja, wir alle wissen, dass dies leichter gesagt als getan ist. Sehr schnell wurde mir bewusst, dass man die anwaltliche Praxis im Referendariat eher weniger lernt. Man weiß zwar, wie eine Klage gefertigt wird, aber nicht, wie man eine korrekte Rechnung stellt. Ähnliche Fragen stellen sich bei der Durchsetzung einer Forderung, sobald ein Titel vorhanden ist, und der Beantragung eines Kostenfestsetzungsbeschlusses. Natürlich waren und sind die Kolleginnen und auch mein Kollege so nett und haben mir all diese Dinge gezeigt und beigebracht. Eine Frage jedoch konnten wir bisher noch nicht zufriedenstellend lösen: Wie reagiert man, wenn eine Kollegin damit droht, einen bei der Rechtsanwaltskammer anzuzeigen? Dies passierte mir tatsächlich im zweiten Monat meiner Tätigkeit. Der Adrenalinspiegel steigt in solchen Momenten ins Unermessliche ... Ich habe lange überlegt, ob ich diesen Punkt hier ansprechen soll oder nicht. Ich entschied mich dafür, um künftigen jungen Kolleginnen die Angst zu nehmen, etwas falsch zu machen. Nichts wird so heiß gegessen, wie es gekocht wird. Offensichtlich hatte die Kollegin einen schlechten Tag, denn sie entschuldigte sich bei mir. Eine Anzeige bei der Kammer erfolgte nicht. $\mathrm{Zu}$ einem späteren Zeitpunkt traf ich die Kollegin übrigens vor Gericht und lernte sie persönlich kennen. Vergessen werde ich die Angelegenheit jedoch sicher nicht so schnell.

Einen weiteren Aspekt meiner Tätigkeit will ich nicht auslassen: die Vertretung und das Auftreten vor Gericht. Bedauerlicherweise hatte ich während des Referendariats nicht die Gelegenheit dazu. Somit musste ich meinen ersten Gerichtstermin vor dem Amtsgericht (in geliehener Robe!) völlig auf mich allein gestellt wahrnehmen. Zugegeben, das muss sich nicht wiederholen. Wird es auch nicht. Mittlerweile bin ich darin etwas erfahrener und somit auch routinierter. Gerichtstermine gehören zum Job, ebenso wie die Beratung von Mandanten und das Fertigen von Schriftsätzen.

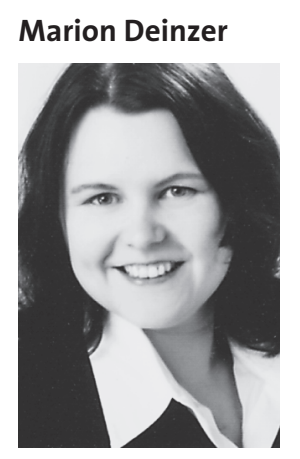

Rechtsanwältin, Fürth 
Mittlerweile ist über ein halbes Jahr vergangen, dass ich richtig in den Beruf und somit in den Anwaltsalltag eingestiegen bin. Die Zulassung habe ich seit fast einem Jahr. Ich erlebte Kurioses, Schönes, Erstaunliches, Unerfreuliches, Kollegiales und auch nicht so Kollegiales. Für mich war es die richtige Entscheidung, ich habe keinen Tag bereut. Das liegt vielleicht daran, dass ich bereits vor dem Studium Rechtsanwältin werden wollte und diesen Berufswunsch nie aufgegeben habe. Etwas anderes gab es für mich nicht. $\mathrm{Ob}$ ich eine gute, mittelmäßige oder gar schlechte Anwältin bin, weiß ich nicht. Das müssen andere beurteilen. Jedenfalls kann ich künftigen Berufsanfängerinnen nur raten, ihren Weg zu gehen und sich nicht entmutigen zu lassen. Wir alle wissen, dass die Arbeitsmarktsituation schlecht ist, kombiniert mit einem schlechten Examen wird es fast aussichtslos. Zumindest wird uns das immer wieder erzählt. Mittlerweile glaube ich aber, dass dem nicht ganz so ist. Man sollte bereit sein, Kompromisse einzugehen und sich einfach darauf einlassen. Vielleicht entwickelt sich dann der Traumjob daraus, das weiß man vorher einfach nicht.

$\mathrm{Ob}$ ich mir den Anwaltsalltag so vorgestellt habe? Ja und nein. Manchmal nenne ich das, was ich tue, den täglichen Wahnsinn, oft aber ist dieser Beruf wirklich sehr schön. Wenn die Versicherung das geltend gemachte Schmerzensgeld zahlt, der Gegner dem Vergleich zustimmt, die Richterin die vorgetragene Rechtsauffassung teilt oder ein kleineres Kind, das einen Unfall hatte, ein Bild malt, weil die Kanzlei die Sache so gut abgewickelt hat, dann weiß man, es war die richtige Entscheidung und die mitunter schwierige und lange Ausbildung hat sich auf jeden Fall gelohnt! Für mich ist mein Beruf ein Traumjob, den ich nicht mehr missen möchte.

\section{Von o auf 100 oder: plötzlich Richterin}

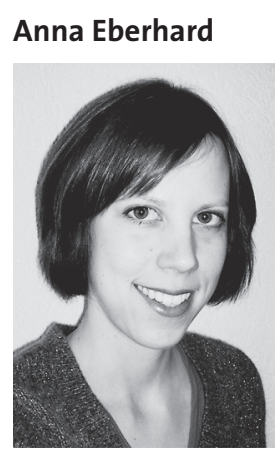

Richterin, Bonn
Während des Studiums und auch noch nach dem ersten Examen wusste ich noch gar nicht richtig, in welchen Beruf mich die Rechtswissenschaften tragen würden. Auch im Laufe des Referendariats konnte ich mich nicht recht entscheiden: allen Stationen konnte ich etwas abgewinnen, die Arbeit war überall neu, spannend und machte mir Spaß. Erst gegen Ende der zwei Jahre merkte ich: eigentlich würdest du gern Richterin werden. Also verbrachte ich die letzten drei Monate des Referendariats am Oberlandesgericht in Köln, um letzte Zweifel zu zerstreuen - mit Erfolg.

Nach dem Examen im November bewarb ich mich am OLG, wurde im Dezember zum Vorstellungsgespräch geladen und erhielt noch am gleichen Tag die Zusage für das Landgericht Köln. Ich habe mich sehr gefreut und konnte kaum glauben, wie schnell mein Berufswunsch in Erfüllung gehen sollte! Nach Erledigung der Formalitäten hatte ich im Februar meinen ersten Arbeitstag. Ich wurde von meinen Kammer-Kollegen begrüßt und eingewiesen und schon hieß es: „Hier sind Ihre Akten, dort Ihr Computer, dann legen Sie mal los".

Und ich legte los. Zunächst nur sehr langsam und mit 100 Fragezeichen an jeder Akte. Bekommt man doch weder an der Uni, noch im Referendariat gesagt, wie Akten verwaltet werden, was man mit Sachverständigengutachten tut, wann man einen Beschluss fertigen muss oder wann eine Verfügung reicht! Zum Glück gab (und gibt) es Kollegen, bei denen ich offene Türen einrannte, wenn ich mit meinem Stapel „Frage-Akten " um Hilfe bat. Nichtsdestotrotz war das zunächst eine sehr frustrierende Arbeit, weil ich das
Gefühl hatte, wegen jeder Akte und jeder neuen „Aufgabe“ fragen zu müssen. Um so schöner der erste Tag, an dem ich alle Akten ohne Hilfe bearbeiten konnte! Wobei sich schon am nächsten Tag wieder Fragen über Fragen stellten ...

In Woche zwei begannen für mich die Sitzungen. Die erste Sitzung war glücklicherweise eine Kammersitzung, bei der ich mir eine Verhandlungsleitung noch einmal „ansehen“ konnte dann aber ging es auch mit Einzelrichtersitzungen los. Das Wochenende vorher hatte ich nicht nur meine Fälle zur Vorbereitung nach Hause mitgenommen, sondern auch das Diktiergerät, um den Umgang damit zu üben. Zu meiner großen Überraschung klappte dann aber alles ganz passabel und die erste Sitzung war schneller vorbei als gedacht!

Zwischen all dem Neuen und Unerwarteten kam dann nach einigen Wochen das erste Urteil. Endlich hatte ich das Gefühl: Urteile schreiben hast du gelernt, das kannst du! Auch wenn inhaltlich natürlich wiederum alles neu und unbekannt und auch nicht immer einfach war.

Meine ersten Wochen als Richterin waren und sind noch immer - sehr anstrengend und die Arbeit nimmt viel (Frei-)Zeit in Anspruch. Wenn ich aber mit meinen Proberichterkollegen spreche, die alle in meiner Situation stecken, höre ich: allen geht es wie mir. Und wenn mir dann eine Sitzung gut gelingt, ich mit meiner Argumentation meine Kammer-Kollegen überzeugen kann oder das Oberlandesgericht eine Entscheidung von mir bestätigt, merke ich, dass der Beruf an sich und ohne die Anfangsschwierigkeiten das Richtige für mich ist! 